\title{
Emerging strategies for the treatment of pulmonary tuberculosis: promise and limitations?
}

\author{
Wing Wai Yew ${ }^{1}$ and Won-Jung Koh ${ }^{2}$
}

\author{
${ }^{1}$ Stanley Ho Centre for Emerging \\ Infectious Diseases, The Chinese \\ University of Hong Kong, Hong \\ Kong; ${ }^{2}$ Division of Pulmonary and \\ Critical Care Medicine, Department of \\ Medicine, Samsung Medical Center, \\ Sungkyunkwan University School of \\ Medicine, Seoul, Korea \\ Received: May 6, 2015 \\ Accepted: May 11, 2015 \\ Correspondence to \\ Wing Wai Yew, M.D. \\ Stanley Ho Centre for Emerging \\ Infectious Diseases, The Chinese \\ University of Hong Kong, Hong \\ Kong \\ Tel: $+852-2252-8884$ \\ Fax: $+852-2635-4977$ \\ E-mail: yewww@cuhk.edu.hk
}

A worsening scenario of drug-resistant tuberculosis has increased the need for new treatment strategies to tackle this worldwide emergency. There is a pressing need to simplify and shorten the current 6-month treatment regimen for drug-susceptible tuberculosis. Rifamycins and fluoroquinolones, as well as several new drugs, are potential candidates under evaluation. At the same time, treatment outcomes of patients with drug-resistant tuberculosis should be improved through optimizing the use of fluoroquinolones, repurposed agents and newly developed drugs. In this context, the safety and tolerance of new therapeutic approaches must be addressed.

Keywords: Tuberculosis; Drug resistance; Therapeutics

\section{INTRODUCTION}

Tuberculosis (TB) is a major infectious disease that causes significant morbidity and mortality $[1,2]$. The principal challenge in its treatment is the emergence of drug-resistant forms leading to worsening scenarios worldwide $[3,4]$. Multidrug-resistant (MDR)-TB is defined as bacillary resistance to isoniazid and rifampicin. Extensively drug-resistant (XDR)-TB is usually defined as MDR-TB with additional bacillary resistance to fluoroquinolones and one or more of the second-line injectable drugs of kanamycin, amikacin and capreomycin. The spread of drug resistance is a cause for concern and totally drug-resistant-TB has been reported in some parts of the world, such as India and South Africa [5-7].

Given this worsening scenario, new strategies to meet the therapeutic challenge of drug-resistant TB would need to achieve: (1) shortening and simplification of the treatment regimens of drug-susceptible TB and (2) an improvement of the treatment of MDR-TB and XDRTB. These strategies can be viewed as preventative, to forestall the emergence of forms of TB that are difficult to treat, and definitive, to tackle the established scenarios of drug resistance, respectively.

To help our understanding, this mini-review is limited to strategies for the treatment of pulmonary TB only; those concerning latent infections due to Mycobacterium tuberculosis are beyond the scope of this review. Human immunodeficiency virus (HIV) coinfection issues are also only mentioned briefly. Furthermore, immunotherapy and other adjunctive treatment options for TB, such as surgery, are not included in this article. 
SHORTENING AND SIMPLIFYING SHORTCOURSE CHEMOTHERAPY FOR PULMONARY TB

\section{The biology of $M$. tuberculosis and the scientific basis of short-course TB treatments}

In theory, there exist different populations of $M$. tuberculosis within pathological TB lesions in the body (commonly in the lungs) of the host, or within different parts of the same lesion. This phenomenon of heterogeneity is highly relevant to our understanding of the scientific basis of short-course chemotherapies used to treat TB $[8,9]$. In general, there are abundant bacilli undergoing continuous growth and rapid replication that constitute a distinct mycobacterial population commonly found in highly aerobic environments, such as the lumen of a pulmonary cavity. Other bacilli undergoing spurts of metabolism or even hibernation are also present. These organisms are known as persisters [10]. Such hibernating mycobacteria develop through stochastic evolutionary processes and are induced by environmental clues such as antibiotic stress and nutrient deprivation, and are believed to reside in unstable pulmonary lesions, such as the biofilms on the inner wall of cavities, or in inflammatory areas in the lung parenchyma, or even intracellularly within macrophages. Unlike the rapidly growing organisms, which are susceptible to bactericidal activity of antimicrobial drugs, the persisters can only be tackled by agents that have a sterilizing activity [9]. In the treatment of TB, drugs with distinct bactericidal activity usually include isoniazid and sometimes also aminoglycosides, whereas drugs that have a predominant sterilizing activity include the rifamycins and the fluoroquinolones (which also have some bactericidal activity). Pyrazinamide, however, only has a sterilizing activity. The totally dormant organisms are assumed to reside largely in densely fibrotic and calcified areas of the lungs, and it is believed that they cannot be readily killed by antimicrobial therapies [10].

\section{Rationale for shortening the current short-course TB chemotherapy}

The conventional or standard short-course treatment for pulmonary TB comprises an intensive phase with rifampicin and isoniazid, as well as pyrazinamide and ethambutol for 2 months, followed by a continuation phase that comprises the concomitant use of rifampicin and isoniazid for another 4 months [11]. Thus, the total duration of therapy lasts for 6 months. A strategy known as directly observed treatment, short-course (DOTS), focusing on supervised therapy and holistic care, is generally recommended to achieve a good patient cure rate, and to curtail the development of bacillary resistance to drugs. However, a 6-month treatment is lengthy and patient adherence throughout can be difficult to guarantee. In addition, the amount of resources required to deliver DOTS, even with enlistment of community participation, can be substantial, especially in countries with a high TB burden. As a result, it is easy to understand why a shorter treatment duration should be investigated as an alternative method to treat TB $[12,13]$.

\section{Possible approaches to shorten drug-susceptible TB chemotherapy and its associated limiting issues}

The most successful and workable approach would be to explore more effective means of dosing and scheduling of currently available agents to enable efficacious and efficient treatment of TB. In this context, two groups of drugs can readily be considered; the rifamycins and the fluoroquinolones. Newly developed drugs for the treatment of TB also offer considerable promise (Fig. 1).

\section{Rifamycins}

Rifamycins have a dose-dependent, early bactericidal activity (EBA) against $M$. tuberculosis, with a linear rela-

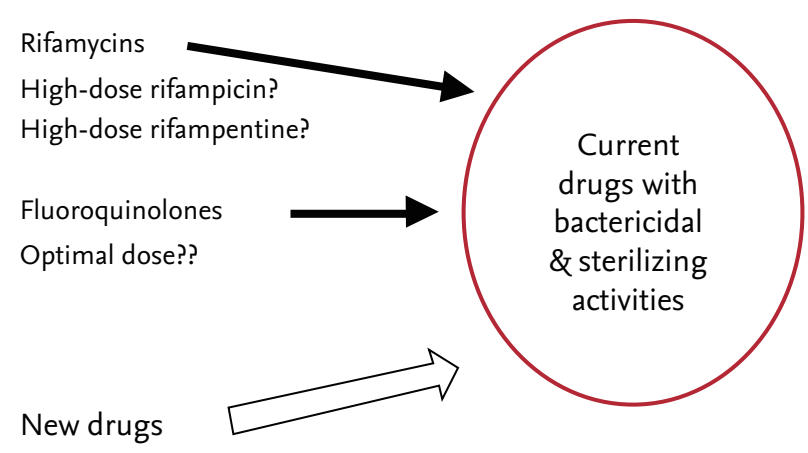

Figure 1. Potential candidates for a reduced 6-month treatment duration of drug-susceptible tuberculosis. 
tionship to a logarithmic dose at lower doses and a curvilinear response at higher doses [14]. Thus, enhancing the rifamycin delivery method by increasing the dose and frequency of administration might boost its activity against $M$. tuberculosis. In addition, rifamycins have a sterilizing activity that can eliminate the mycobacterial persisters (mainly semi-dormant or near-dormant organisms) within the host [15]. This anti-persister mechanism is highly relevant in attempts to shorten the duration of TB treatment, as the persisters are generally regarded as the culprits of relapses of TB disease [16].

A randomized controlled trial (RCT) on drug-susceptible TB, involving patients treated with rifapentine, a long-acting cyclopentyl rifamycin, at a dose of $10 \mathrm{mg} /$ $\mathrm{kg}$ versus rifampicin at a dose of $10 \mathrm{mg} / \mathrm{kg}$, both for 5 days per week, did not reveal significantly better activity of the former over the latter with respect to an 8-week sputum culture conversion from positive to negative, although the longer-acting rifapentine was found to be safe and was well-tolerated at the explored dose and frequency of administration [17]. Higher rifapentine exposure (20 mg/kg daily, for 7 days per week) was significantly associated with a higher sputum culture conversion from positive to negative on completion of an 8-week intensive phase of treatment [18]. Greater exposure to rifapentine, via a higher dosage, was also tolerated by patients. Two other RCTs of rifapentine-based regimens are awaiting analysis of the results. One of the trials compares rifapentine $600 \mathrm{mg} / 450 \mathrm{mg}$ versus rifampicin $600 \mathrm{mg}$ (NCToo814671), and the other compares rifapentine $7.5 \mathrm{mg} / \mathrm{kg}$ and moxifloxacin versus rifampicin $10 \mathrm{mg} / \mathrm{kg}$ and ethambutol (NCT 00728507).

The results of an EBA study on the use of high-dose rifampicin have also been published recently [19]. In this study, patients with drug-susceptible TB were initially enrolled into a control group receiving the standard rifampicin dose of $10 \mathrm{mg} / \mathrm{kg}$, whereas the experimental groups each received 20, 25, 30, and $35 \mathrm{mg} /$ $\mathrm{kg}$, respectively, for 14 days. Over the last 7 days, each patient also received isoniazid, ethambutol, and pyrazinamide in the usual doses. Peak serum rifampicin levels and total rifampicin exposure showed a higher than proportional escalation with higher doses and a larger than estimated reduction in the bacillary load in the two highest dose groups. Safety and tolerance were within normal levels for all doses, but the study duration and number of subjects were limiting factors and did not enable complete confidence in the toxicity assessment. In a case-control study in India, a peak plasma rifampicin concentration of $12.5 \mathrm{mg} / \mathrm{L}$ or more on day 7 possibly predicted the subsequent development of drug-induced hepatitis in the vast majority of patients [20]. Other high-dose rifampicin studies are listed in Table 1. Some of these have already been completed and the results are currently being analyzed. The reports are awaited with great interest.

Furthermore, while all these enhanced rifamycin trials have alluded to greater efficacy in terms of enhanced bactericidal activity in the early weeks of treatment (with reduced culture times and an increased proportion of subjects showing bacteriological improvement), the potential for these parameters to act as robust markers for sterilizing activity remains contentious. The relapse rate on follow up after completion of the full course of TB treatment remains the gold standard to evaluate the sterilizing activity of a drug/drug regimen and the potential of the agent(s) to shorten the length of the TB treatment [21]. Improved understanding of $M$. tuberculosis persister will increase the potential to discover biomarkers better suited to identify viable, nonculturable mycobacteria and, thus, assess

Table 1. Clinical trials of high-dose rifampicin in the treatment of drug-susceptible tuberculosis

\begin{tabular}{lll}
\hline Trial registration number & \multicolumn{1}{c}{ Trial drug regimen } & Trial phase \\
\hline ISRCTN 55670677 & Rifampicin: $20 \mathrm{mg} / \mathrm{kg}$ vs. $15 \mathrm{mg} / \mathrm{kg}$ vs. $10 \mathrm{mg} / \mathrm{kg}$ & IIB \\
NCTo0760149 & Rifampicin: $1,200 \mathrm{mg}$ vs. $900 \mathrm{mg}$ vs. $600 \mathrm{mg}$ & IIB \\
NCTo1408914 & Rifampicin: $20 \mathrm{mg} / \mathrm{kg}$ vs. $15 \mathrm{mg} / \mathrm{kg}$ vs. $10 \mathrm{mg} / \mathrm{kg}$ & IIB \\
NCTo1785186 & Rifampicin $35 \mathrm{mg} / \mathrm{kg}+$ Ethambutol vs. Rifampicin 10 mg/kg + SQ109 vs. & IIB \\
& Rifampicin $20 \mathrm{mg} / \mathrm{kg}+$ SQ109 vs. Rifampicin $20 \mathrm{mg} / \mathrm{kg}+$ Moxifloxacin vs. & \\
& Rifampicin $10 \mathrm{mg} / \mathrm{kg}+$ Ethambutol & \\
\hline
\end{tabular}


the chances of relapse from the proportion of mycobacterial persisters, during and after short-course TB treatments $[22,23]$. Possible approaches, phenotypic and transcriptomic in nature, are now under active investigation $[24,25]$.

\section{Fluoroquinolones}

Prompted by the encouraging results from murine TB models of the possible sterilizing effects of later generation fluoroquinolones $[26,27]$, a number of studies, many of which are RCTs [28-36], have aimed to identify whether fluoroquinolones could be used to shorten

Table 2. Clinical trials on the use of fluoroquinolones in drug-susceptible tuberculosis

\begin{tabular}{|c|c|c|}
\hline Source & Study design & Findings summary \\
\hline $\begin{array}{l}\text { Burman et al. } \\
(2006)[28]\end{array}$ & RCT & $\begin{array}{l}\text { Substituting moxifloxacin for ethambutol during the intensive phase of } \\
\text { treatment did not affect the } 2 \text {-month sputum culture status (71\% in both groups), } \\
\text { but did increase the proportion of negative cultures at earlier time points. } \\
\text { Patients treated with moxifloxacin more commonly suffered from nausea, but } \\
\text { similar proportions in both groups completed the study treatment ( } 88 \% \text { vs. } 89 \% \text { ). }\end{array}$ \\
\hline $\begin{array}{l}\text { Dorman et al. } \\
(2009)[29]\end{array}$ & RCT & $\begin{array}{l}\text { Substituting moxifloxacin for isoniazid during an intensive phase of treatment } \\
\text { resulted in a small but nonsignificant increase in culture negativity after } 8 \\
\text { weeks (60.4\% vs. } 54.9 \%) \text {. }\end{array}$ \\
\hline $\begin{array}{l}\text { Wang et al. } \\
(2010)[30]\end{array}$ & Cohort study & $\begin{array}{l}\text { Adding moxifloxacin to isoniazid, rifampicin, pyrazinamide and ethambutol } \\
\text { shortened the time to culture conversion, and significantly increased the } \\
\text { proportion of culture conversion after } 6 \text { weeks ( } 82 \% \text { vs. } 61 \%) \text {. }\end{array}$ \\
\hline $\begin{array}{l}\text { Conde et al. } \\
(2009)[31]\end{array}$ & $\mathrm{RCT}$ & $\begin{array}{l}\text { Adding moxifloxacin instead of ethambutol to isoniazid, rifampicin and } \\
\text { pyrazinamide significantly improved the proportion of converted cultures after } \\
8 \text { weeks ( } 80 \% \text { vs. 63\%). }\end{array}$ \\
\hline $\begin{array}{l}\text { Jawahar et al. } \\
(2013)[32]\end{array}$ & $\mathrm{RCT}$ & $\begin{array}{l}\text { Administering gatifloxacin or moxifloxacin instead of ethambutol in the first } \\
2 \text { months, with isoniazid, rifampicin and pyrazinamide for } 6 \text { months } \\
\text { (thrice weekly) resulted in significantly more recurrences in the gatifloxacin } \\
\text { group than the control group ( } 15 \% \text { vs. } 6 \% \text { ). }\end{array}$ \\
\hline $\begin{array}{l}\text { Gillespie et al. } \\
(2014)[33]\end{array}$ & $\mathrm{RCT}$ & $\begin{array}{l}\text { Substituting moxifloxacin for either ethambutol or isoniazid in the first } 17 \\
\text { weeks followed by a placebo for } 8 \text { weeks resulted in significantly more unfavorable } \\
\text { outcomes (treatment failure or relapse within } 18 \text { months) in a } 4 \text {-month regimen } \\
\text { compared to the standard 6-month regimen (difference: } 11.4 \% \text { and } 6.1 \% \text {, respectively). }\end{array}$ \\
\hline $\begin{array}{l}\text { Merle et al. } \\
(2014)[34]\end{array}$ & RCT & $\begin{array}{l}\text { A 4-month regimen comprising isoniazid, rifampicin, and gatifloxacin } \\
\text { supplemented by pyrazinamide during a 2-month intensive phase yielded } \\
\text { significantly more unfavorable outcomes (treatment failure, recurrence, death, } \\
\text { or dropout during treatment) than the standard 6-month regimen (difference: } 3.5 \% \text { ). } \\
\text { Recurrence rates of the 4- and 6-month regimens were } 14.6 \% \text { vs. } 7.1 \% \text {, respectively). }\end{array}$ \\
\hline $\begin{array}{l}\text { Jindani et al. } \\
(2014)[35]\end{array}$ & RCT & $\begin{array}{l}\text { A 4-month daily regimen comprising moxifloxacin, rifampicin, pyrazinamide } \\
\text { and ethambutol in the first } 2 \text { months followed by moxifloxacin and rifapentine } \\
\text { twice weekly for } 2 \text { months was compared with a } 6 \text {-month regimen } \\
\text { (continuation phase: moxifloxacin and rifapentine weekly for } 4 \text { months) and } \\
\text { a } 6 \text {-month standard regimen. The } 4 \text {-month moxifloxacin-based regimen } \\
\text { resulted in significantly more unfavorable responses (treatment failure or } \\
\text { relapse), with a difference of } 13.6 \% \text { per protocol analysis and } 13.1 \% \text { by modified } \\
\text { intention-to-treat analysis. The } 6 \text {-month moxifloxacin-based regimen was found } \\
\text { to be as effective as the } 6 \text {-month standard regimen. }\end{array}$ \\
\hline $\begin{array}{l}\text { Velayutham et al. } \\
(2014)[36]\end{array}$ & RCT & $\begin{array}{l}\text { A daily regimen comprising moxifloxacin, isoniazid, rifampicin, ethambutol, } \\
\text { and pyrazinamide yielded a significantly higher proportion of culture conversion } \\
\text { after } 2 \text { months of treatment ( } 95 \% \text { vs. } 81 \% \text { ). }\end{array}$ \\
\hline
\end{tabular}

RCT, randomized controlled trial. 
the duration of TB chemotherapy. These studies are listed in Table 2. For example, one RCT suggested that moxifloxacin might have sterilizing activity due to the rate of conversion of a sputum culture from positive to negative after 8 weeks of treatment [31]; however, the relapse rate after cessation of treatment would reflect the sterilizing capacity of the drug/drug regimen more accurately. Currently, 4-month moxifloxacin-/gatifloxacin-containing regimens have not been shown to be non-inferior to the standard 6-month short-course regimen currently in use [33-35]. Although one RCT suggested that adding moxifloxacin to the standard 4-drug short-course regimen would enhance the bactericidal activity of the combination substantially, interpretation of the results might be limited by the confounding difference in the dosing schedules between the test regimens and the control regimen [36]. It is possible that the potential sterilizing activity of the later generation fluoroquinolones is related to the size of the dose, similar to the rifamycins. More work is needed to explore this, and the safety and tolerance of these compounds with dose escalation must also be evaluated.

New drugs

Several new drugs, including bedaquiline (a diaryquinoline that interferes with ATP synthesis), delamanid (a nitro-dihydro-imidazooxazole derivative that inhibits mycolic acid biosynthesis), pretomanid or PA-824 (a nitroimidazo-oxazine with a similar mechanism of action to delamanid), and sutezolid (an oxazolidinone that acts on ribosomal protein synthesis) have all completed evaluation in phase II clinical trials for efficacy and safety as anti-TB drugs.

Bedaquiline has significant EBA at a dose of 400 mg [37]. Another 14-day EBA study selected patients with drug-susceptible TB randomly to receive combinations of bedaquiline with pyrazinamide-clofazimine, pretomanid-pyrazinamide, pretomanid-pyrazinamide-clofazimine and pretomanid-clofazimine, or clofazimine, or pyrazinamide alone, or the standard 4-drug combination [38]. The combination of bedaquiline-pretomanid-pyrazinamide yielded the most favorable activity. It should be noted that two newly developed drugs were included in this novel regimen.
A randomized dose-ranging study regarding the EBA of delamanid at 100, 200,300, and $400 \mathrm{mg}$ over 14 days revealed exposure-dependent activity [39]. The drug exposure, however, plateaued at $300 \mathrm{mg}$, presumably due to absorption limitations resulting in less than proportional exposure increases.

A 14-day EBA study randomized patients to receive $200,600,1,000$, and 1,200 $\mathrm{mg}$ of pretomanid and the results indicated an optimal performance at the relatively low dose of $200 \mathrm{mg}$ [40]. Another randomized dose-ranging phase II study of 14-day EBA of a once-daily pretomanid dose at 50, 100, 150, and 200 mg demonstrated acceptable safety and tolerance at all doses [41]. A lower EBA was observed in those patients given pretomanid $50 \mathrm{mg}$ once daily, but this was not statistically significant. A further phase IIA EBA study evaluated patients randomized to receive 5-drug combinations in comparison with the standard 4-drug shortcourse regimen, i.e., pretomanid with bedaquiline, pretomanid with pyrazinamide, pretomanid with moxifloxacin and pyrazinamide, bedaquiline, and bedaquiline with pyrazinamide [42]. The dosages of pretomanid and bedaquiline were 200 and $400 \mathrm{mg}$ once daily, respectively. The 14-day EBA of pretomanid with moxifloxacin and pyrazinamide was comparable with those of pretomanid with pyrazinamide and the standard 4-drug combination, and was significantly higher than the other groups. The favorable results shown by this 3-drug combination have been corroborated by a subsequent phase IIB open-label, partly- randomized study that compared the bactericidal activity of the combination of pretomanid, moxifloxacin, and pyrazinamide with that of rifampicin, isoniazid, ethambutol, and pyrazinamide, which demonstrated the superiority of the former regimen [43]. Thus, the combination of pretomanid, moxifloxacin, and pyrazinamide could be useful for treating TB, and a phase III study is now underway (STAND trial: GATB NC-oo6).

Sutezolid is a newer member of the oxazolidinones, of which linezolid is a known repurposed drug used in the treatment of TB. Repurposed drugs are agents that were initially not intended for the treatment of TB when launched. A 14-day EBA study of sutezolid in the sputum, as well as an assessment of intracellular bactericidal effects using whole blood cultures, showed favorable results when the drug was utilized at 
a dosage of $600 \mathrm{mg}$ twice daily or 1,200 mg once daily [44]. AZD5847, another member of this class of compounds, has been assessed in an extended EBA study (NCTo1516203) involving a dose-ranging evaluation in comparison with the standard rifampicin-isoniazid-ethambutol-pyrazinamide combination. The results are currently being analyzed.

As bedaquiline, delamanid and pretomanid all have proven sterilizing activity, as demonstrated in animal models, there is the distinct possibility that these compounds can be included in the design of novel drug regimens to shorten current TB chemotherapies [45]. The 14-day EBA (phase IIA)/phase IIB studies can help to demonstrate the bactericidal activity of these agents and possibly shed some light on their sterilizing activities against mycobacterial persisters [46]. One major issue with regard to the long-term use of these new drugs in a clinical setting concerns the limited amount of data regarding their safety and tolerance. This could be addressed through evaluations in phase III trials [45]. Likewise, the newer oxazolidinone congeners, sutezolid and AZD 5847, which have potential roles for treating drug-susceptible and drug-resistant forms of TB, also face similar problems.

The rising resistance rates of $M$. tuberculosis strains to fluoroquinolones and pyrazinamide, especially in patients with acquired MDR-TB, but also in those with a high risk of transmitted bacillary resistance, pose possible limitations on the use of these "new" drug regimens [47]. Clearly any adverse bacillary resistance would hamper the efficacy of such regimens concomitantly containing fluoroquinolones and pyrazinamide, which are also intended to contribute to the sterilizing activity of these novel treatment regimen options [45].

Drug-drug interaction is another hurdle in the development of regimens to shorten the duration of TB chemotherapy. The interaction between rifampicin and moxifloxacin, possibly through induction of effects of the former on the glucuronidation or sulphation of the latter, was shown to result in reduced plasma concentrations of fluoroquinolone [48]. In a study to evaluate the safety, tolerability, pharmacokinetics and bacteriological effects of different doses of SQ109 (an ethylenediamine derivative analogous to ethambutol), a $300 \mathrm{mg}$ dose yielded a higher exposure than the 150mg dose upon coadministration of rifampicin. SQ109 did not appear to be active on its own or to enhance the activity of rifamycin [49]. In a similar fashion, as bedaquiline constitutes a substrate for the cytochrome P45o isozymes (isoenzymes), expected interactions with inducers such as rifampicin would occur and might lead to attenuation of the therapeutic activity of the diarylquinoline [50]. Some TB patients also suffer from HIV coinfection, and in these cases the potential for complex pharmacokinetic and pharmacodynamic interactions between antiretroviral drugs and new anti-TB drugs can be daunting. Overlapping toxicities, notably dermatopathy, neurotoxicity, cardiotoxicity, nephrotoxicity, and other adverse effects might also pose a significant challenge in the clinical management of these patients [45]. Aside from HIV infection, other populations also vulnerable to drug-drug interactions include elderly patients and those with organ transplants [51].

Bedaquiline is a substrate of cytochrome $\mathrm{P}_{450}$ (CYP) $3 \mathrm{~A}_{4}$, and is actively metabolized to a N-monodesmethyl derivative. As mentioned above, the rifampicin-bedaquiline interaction hampers the development of diarylquinoline for the treatment of drug-susceptible TB when rifamycin is included in the drug regimen. Interaction of bedaquiline with efavirenz, a non-nucleoside reverse transcriptase inhibitor, has been reported, albeit with some variability in the severity of the outcomes $[52,53]$. Bedaquiline should only be administered with caution to patients receiving ritonavir-boosted lopinavir, as there would be a resultant escalation of bedaquiline exposure due to pharmacokinetic inhibition [54]. An estimated 30\% of sutezolid and $20 \%$ of pretomanid doses are also metabolized by $\mathrm{CYP}_{3} \mathrm{~A}_{4}$ [55]. Efavirenz reduced the exposure of pretomanid by $\sim 30 \%$, but lopinavir-ritonavir exerted much less effect [56]. Delamanid has no known association with the CYP isozymes and; thus, has a low potential for pharmacokinetic interactions with this family of isozymes [57]. The metabolism of SQ109 is not associated with $\mathrm{CYP}_{3} \mathrm{~A}_{4}$ but this ethylenediamine is a substrate of $\mathrm{CYP}_{2} \mathrm{D} 6$ and $\mathrm{CYP}_{2} \mathrm{C}_{1}$, which can be significantly inhibited by some triazoles, albeit with uncertain clinical implications [55,58].

In the future, as novel regimens for the treatment of TB may include more than one newly developed agent, thorough study is needed of their interactions in the 
context of efficacy and toxicity. At present there are limited data regarding the favorable interactions between SQ109 and bedaquiline with respect to the efficacy of both agents [59]. Interactions between pretomanid and bedaquiline, as well as between delamanid and bedaquiline, especially in terms of safety and tolerability, also require further investigation. A study for the latter is soon to be launched (ACTG A5343).

\section{IMPROVING THE TREATMENT OF MDR-TB AND XDR-TB}

There are two major strategies to capitalize on the current tools available to treat MDR-TB and XDR-TB. These are (1) optimizing the use of later generation fluoroquinolones, and (2) review the use of repurposed agents. In the face of the suboptimal treatment success rates of MDR-TB in many countries worldwide [6o], there is clearly a genuine need for newly-developed drugs/drug regimens to stop the looming epidemic of XDR-TB, which develops through both acquired and transmitted resistance mechanisms $[7,61]$.

\section{Possible new approaches to improving the out- comes of drug-resistant TB}

High-dose fluoroquinolones

There is unequivocal evidence that the fluoroquinolones are pivotal agents in the treatment of drug-resistant TB. The later generation fluoroquinolones are likely to have greater efficacy compared to the earlier agents, especially for use in fluoroquinolone-resistant MDR-TB and XDR-TB [62]. The Bangladesh regimen is based on the use of high-dose gatifloxacin, up to 800 mg once daily, alongside clofazimine, ethambutol, and pyrazinamide taken over 9 months and supplemented by kanamycin, prothionamide, and a moderately high dose of isoniazid ( $10 \mathrm{mg} / \mathrm{kg} /$ day $)$ for a minimum of 4 months; this regimen has achieved a relapse-free cure rate of $88 \%$ in patients with MDR-TB who were previously untreated with second-line drugs [63]. The remarkable efficacy of this shortened MDR-TB treatment regimen, compared with the 20-month regimes, as recommended by World Health Organization [62], might attest to the dose-dependent bactericidal and steriliz- ing activities of the later generation fluoroquinolones. In addition, the high dose would also lessen the risk of the development of fluoroquinolone resistance in $M$. tuberculosis strains. As the Bangladesh regimen also incorporates other drugs, there has been much enthusiasm in attributing the efficacy of the regimen to these accompanying agents, especially clofazimine [64-66]. Table 3 summarizes the pertinent findings from these studies.

However, the good long-term treatment outcomes in this observational cohort of patients, except among those with bacillary resistance to fluoroquinolone at phenotypically high levels, affirms the key therapeutic role of the high-dose fluoroquinolone in the Bangladesh regimen [67]. The regimen also highlights the paramount importance of programmed management of MDR-TB to minimize the development of bacillary resistance to fluoroquinolones, alongside the prudent use of short-course fluoroquinolones in the treatment of bacterial infections, especially those pertaining to the lower respiratory tract [68].

More importantly, tolerance to the Bangladesh regimen was remarkable [63]. Less than $10 \%$ of patients experienced major adverse reactions, and this included dysglycemic reactions in diabetic subjects. Unfortunately, due to a dwindling supply of gatifloxacin worldwide, the efficacy and safety of other, later generation fluoroquinolones that can be used at high dosages must be explored, despite the associated increased costs. Currently, there are two ongoing studies; the STREAM trial, which is evaluating moxifloxacin [69], and the Opti-Q trial, which is evaluating levofloxacin (NCTo1918397). The STREAM trial has recently included an additional section to evaluate the benefit and safety of incorporating bedaquiline, but the fluoroquinolone used in this case is levofloxacin. Careful evaluation of the safety of different later generation fluoroquinolones is clearly justified, as the risk of adverse reactions, especially cardiotoxicity [45], might differ substantially among these later congeners. The potential pharmacodynamic interaction of bedaquiline and high-dose fluoroquinolone, specifically the additive risks of cardiotoxicity, are discussed below.

\section{Repurposed agents}

The principal agents considered for the management 
Table 3. Recent studies of clofazimine in drug-resistant tuberculosis

\begin{tabular}{|c|c|c|}
\hline Source & Study design & Findings summary \\
\hline Grosset et al. (2013) [64] & Animal study & $\begin{array}{l}\text { Mice infected with isoniazid-resistant } \mathrm{M} \text { tuberculosis were treated with } \\
\text { a combination of moxifloxacin, ethambutol, pyrazinamide, and amikacin. } \\
\text { Half of the mice also received clofazimine. The decline in lung bacillary load } \\
\text { after } 2 \text { months was significantly better in animals in the clofazimine group, } \\
\text { with culture negativity by } 5 \text { months, whereas mice in the other group } \\
\text { remained culture-positive throughout the entire } 9 \text { months of the study. } \\
\text { The relapse rate was only } 7 \% \text { among mice treated with clofazimine. }\end{array}$ \\
\hline Padayatchi et al. (2014) [65] & $\begin{array}{l}\text { Retrospective } \\
\text { cohort study }\end{array}$ & $\begin{array}{l}\text { Patients were treated with either a clofazimine- or non-clofazimine-contain } \\
\text { ing extensively drug-resistant tuberculosis treatment regimen. Most patients } \\
(86 \%) \text { were human immunodeficiency virus-infected and receiving } \\
\text { antiretrovirals ( } 90 \%) \text {. In the clofazimine- and non-clofazimine groups, } \\
40 \% \text { and } 28.6 \% \text { had culture conversion to negativity, respectively. The hazard } \\
\text { rate ratio of conversion at } 6 \text { months was } 2.54 \text { for the clofazimine group. } \\
\text { Adverse reactions due to clofazimine were minor and rarely life threatening. }\end{array}$ \\
\hline Tang et al. (2015) [66] & $\begin{array}{l}\text { Randomized } \\
\text { controlled trial }\end{array}$ & $\begin{array}{l}\text { Patients were randomized to either a clofazimine or a control group, and } \\
\text { received } 21 \text { months of individualized treatment regimens based on their } \\
\text { medication history and drug susceptibility testing results. Sputum culture } \\
\text { conversion was earlier in the clofazimine group than in the control group, and } \\
\text { cavitary closure was also earlier. The treatment success rate in the clofazimine } \\
\text { group was } 73.6 \% \text {, which was significantly higher than the } 53.8 \% \text { in the contro } \\
\text { lgroup, but there was no significant difference in cure rates between the two } \\
\text { groups. Skin discoloration and ichthyosis were common in the clofazimine } \\
\text { recipients. }\end{array}$ \\
\hline
\end{tabular}

of drug-resistant TB largely include linezolid, clofazimine, and meropenem-clavulanate [70]. Two systematic reviews have suggested the importance of linezolid in the treatment of MDR-TB, highlighting the benefit of a lower dose of $600 \mathrm{mg}$ daily with an equivalent efficacy, in light of the substantial and serious hematological and neurological toxicities (over $50 \%$ in total) among recipients of linezolid therapy [71,72]. However, a lower dose, whilst effective in reducing bone marrow toxicity, remains associated with a substantial risk of peripheral neuropathy. A further lowering of the daily dose of linezolid to $300 \mathrm{mg}$ cut the incidence of peripheral neuropathy to $\sim 27 \%[73,74]$. The use of linezolid intermittently three-times a week during the continuation phase after an initial 2 to 3 months of an intensive phase of daily dosing could possibly reduce the risk of peripheral neuropathy, even over prolonged administration, as is often necessary in complicated cases of MDR-TB and XDR-TB [75]. Research is needed to find the optimal balance between efficacy and toxicity for the use of linezolid in the treatment of MDR-TB and XDR-TB. In a systematic review of cohort- and meta-analyses of patients with fluoroquinolone-resistant MDR-TB and XDR-TB, linezolid use significantly increased the probability of a favorable outcome (culture conversion from positive to negative, cure, completion without death, default, failure, or relapse) by $57 \%$ [76]. The contribution of clofazimine and meropenem-clavulanate in the management of these difficult drug-resistance scenarios, independent of that of linezolid, require confirmation [77-80]. However, an underestimation of the add-on benefits of these two repurposed agents is likely to be due to the limited data available and selection bias, as the study [76] was concerned only with a highly selective group of difficult TB patients with formidable bacillary resistance. The role of a high-dose of isoniazid in the management of MDR-TB patients also needs further clarification [76].

\section{New drugs}

A phase IIB RCT has shown that addition of bedaquiline to an optimized background regimen shortened the length of time for sputum culture conversion from positive to negative and improved the proportion of patients with converted cultures, compared with a placebo group, after 2 months of therapy (48\% vs. $9 \%$ ), 
and at 24 weeks during follow up (79\% vs. 58\%) [81,82]. Incorporation of bedaquiline also helped to prevent the development of bacillary resistance to the accompanying drugs in the regimen [82]. A similar RCT of 6-months bedaquiline treatment showed that the addition of this diarylquinoline reduced the time to culture conversion (125 days vs. 83 days), and increased the proportion of culture conversion at 24 (79\% vs. 58\%) and 120 weeks (62\% vs. $44 \%$ ), as well as significantly improved the cure rate at 120 weeks (58\% vs. $32 \%$ ) [83]. However, of concern was the 5 -fold increase in mortality of patients receiving bedaquiline, compared to those who were not [83]. The cause of these deaths requires in-depth investigation, as bedaquiline is known to prolong the QT interval in some patients [84].

A phase IIB RCT of delamanid, administered at 100 and $200 \mathrm{mg}$ twice daily to treat MDR-TB, showed a higher proportion of 2-month sputum culture conversion in those on the new drug $(45.4 \%$ and $41.9 \%$ vs. $29.6 \%$, for low-dose and high-dose delamanid, respectively vs. the placebo) [85]. In a cohort of patients subjected to open-label evaluation, a favorable outcome occurred in $74.5 \%$ of patients administered with at least 6 months of delamanid, but in only $55 \%$ of those who had taken the drug for 2 months [86]. There was also a significant reduction in the mortality rate with longer use compared to the shorter duration of administration: $2.9 \%$ vs. $12.0 \%$ [87]. However, QT prolongation on electrocardiograms was also significantly more frequent among the delamanid users, although without any adverse clinical events [85]. A phase III multicentre RCT of delamanid in MDR-TB (NCTo1424670) has recently been completed and the results are being analyzed. In this study, delamanid was used at $100 \mathrm{mg}$ twice daily for 2 months, followed by $200 \mathrm{mg}$ daily for 4 months, and results on its safety and tolerability data are keenly anticipated.

Pretomanid-moxifloxacin-pyrazinamide appears to be a potentially promising combination for the treatment of drug-susceptible TB $[42,43]$. Although the regimen was also found to be efficacious in a small number of MDR-TB patients [43], the expected compromise in regimen efficacy in the presence of bacillary resistance to fluoroquinolones and pyrazinamide, which can be a genuine issue in some MDR-TB settings [47,67], warrants further investigation.
A 14-day RCT on EBA showed that bedaquiline-pretomanid-pyrazinamide had substantial activity [38]. However, a phase IIB/phase III RCT is required to confirm the clinical efficacy of this regimen, given the limitations of using EBA to fully evaluate the sterilizing activity of drugs. This regimen, using these two new agents, shows potential for the treatment of MDRTB patients. A further study is now underway that incorporates moxifloxacin into the bedaquiline-pretomanid-pyrazinamide combination described above (GATB NC-005).

Finally, the NExT trial will soon be launched in South Africa. This study will evaluate a short-course (6 to 9 months) regimen of bedaquiline, linezolid, levofloxacin, ethionamide, or high-dose isoniazid, and pyrazinamide for the treatment of MDR-TB. In this case, the drug regimen includes the strategic "big three," i.e., a fluoroquinolone, a repurposed agent and a new drug. As the regimen is entirely based on oral drugs, it would be particularly suitable for treating patients with HIV coinfection.

\section{Drug interaction and toxicity}

Although pharmacokinetic interactions through cytochrome $\mathrm{P} 450$ isozymes might occur with a lower frequency in drugs used to treat drug-resistant TB, the possibility of interactive toxicity of a pharmacodynamic nature cannot be dismissed. Cardiototoxicity, due to its potential for fatality, is of particular concern [45]. High-dose fluoroquinolones as well as newly developed drugs, such as bedaquiline and delamanid, could have additive risks for such toxicity with coadministration. The potential risk of cardiotoxicity associated with the use of clofazimine cannot be completely ignored.

Potential overlapping drug toxicities in patients with HIV-MDR-TB, related to the use of antiretrovirals and the anti-MDR-TB agents, have been reported previously [88]. Examples include gastrointestinal reactions, neurotoxicity and metabolic disturbances. In addition, there might be adverse effects related to organ dysfunction, such as nephropathy, as well as immune reconstitution inflammatory syndrome and pharmacokinetic or pharmacodynamic drug-drug interactions in an HIV-infected population. Among the latter type of interactions, nephrotoxicity and peripheral neuropathy are of particular concern. The need for therapeutic 
drug monitoring on an individualized basis, to optimize efficacy and monitor the toxicity of treatment in these HIV/MDR-TB coinfected patients warrants further evaluation $[89,90]$.

\section{CONCLUSIONS}

In the search for, and use of, new drugs to fight TB, the key priorities are to benefit and protect the patients, and also to protect the drugs [91]. Drug safety and tolerability have been widely discussed. Pharmaco-vigilance must be in place at many different levels during the administration of these drugs, especially the newly developed agents. The issue is particularly relevant in the context of the treatment duration of TB, which generally lasts for many months.

Regarding the issue of the emergence of drug resistance in repurposed agents and new drugs, prevention is of paramount importance and; therefore, clear and stringent regulatory guidance cannot be overemphasized. The deterioration of bacillary susceptibility of M. tuberculosis to linezolid is of concern [92,93], especially with the likely increase in the use of this agent for the management of increased drug resistance in many parts of the world [74]. It is very likely that M. tuberculosis can develop a cross-resistance to members of the oxazolidinone class; thus, potentially hampering the activity of emerging congeners, such as sutezolid and AZD 5847. The recent discovery of cross-resistance between clofazimine and bedaquiline, especially among clinical isolates, is alarming [94-96]. However, the underlying mechanism of the efflux pump in exporting the drugs could be amendable to adjuvant therapeutic agents such as verapamil that antagonizes the extrusion of bedaquiline and clofazimine from the mycobacteria, resulting in improved bactericidal activity and suppression of the development of resistance against the drugs $[97,98]$. Although bacillary resistance to delamanid and pretomanid has not been shown to date, the potential for cross-resistance to these nitroimidazole derivatives could have disturbing consequences.

Recently there has been renewed interest in exploring the use of inhaled TB therapies [45]. The rationale is to achieve a higher local concentration of the drugs in the lungs without concomitantly raising the systemic bio- availability that might inadvertently lead to undesirable adverse reactions. This strategy is of greatest relevance in drug-resistant TB settings. There have been some preliminary results in the use of aminoglycosides and the other injectables [99], fluoroquinolones [100] and riminophenazines [101], as well as pretomanid, which is a new agent [102]. It might also be pertinent to explore the potential use of the inhaled route as an adjunct to oral therapy, when the use of orally administered drugs at a high dose is compromised by systemic toxicity. It may be an option to explore such enhanced therapies with later generation fluoroquinolones [45].

The coming decade will probably witness an exciting period of development of new strategies in the treatment of pulmonary TB. This new era in fighting the disease will hopefully be realized through a rational exploration of the limitations of these new therapeutic agents.

\section{Conflict of interest}

WWY is a consultant to Otsuka Pharmaceutical Co. Ltd.

\section{REFERENCES}

1. World Health Organization. Global tuberculosis report 2014 [Internet]. Geneva: World Health Organization, c2015 [cited 2015 Nov 3]. Available from: http://www.who. int/tb/publications/global_report/en/.

2. Glaziou P, Falzon D, Floyd K, Raviglione M. Global epidemiology of tuberculosis. Semin Respir Crit Care Med 2013;34:3-16.

3. Chang KC, Yew WW. Management of difficult multidrug-resistant tuberculosis and extensively drug-resistant tuberculosis: update 2012. Respirology 2013;18:8-21.

4. Falzon D, Mirzayev F, Wares F, et al. Multidrug-resistant tuberculosis around the world: what progress has been made? Eur Respir J 2015;45:150-160.

5. Udwadia ZF, Amale RA, Ajbani KK, Rodrigues C. Totally drug-resistant tuberculosis in India. Clin Infect Dis 2012;54:579-581.

6. Klopper M, Warren RM, Hayes C, et al. Emergence and spread of extensively and totally drug-resistant tuberculosis, South Africa. Emerg Infect Dis 2013;19:449-455.

7. Dheda K, Gumbo T, Gandhi NR, et al. Global control 
of tuberculosis: from extensively drug-resistant to untreatable tuberculosis. Lancet Respir Med 2014;2:321338.

8. Fox W, Mitchison DA. Short-course chemotherapy for tuberculosis. Lancet 1976;2:1349-1350.

9. Mitchison D, Davies G. The chemotherapy of tuberculosis: past, present and future. Int J Tuberc Lung Dis 2012;16:724-732.

10. Zhang Y, Yew WW, Barer MR. Targeting persisters for tuberculosis control. Antimicrob Agents Chemother 2012;56:2223-2230.

11. World Health Organization. Treatment of tuberculosis: guidelines for national programmes (4th edition) [Internet]. Geneva: World Health Organization, c2015 [cited 2015 Nov 3]. Available from: http://www.who.int/tb/publications/tb_treatmentguidelines/en/.

12. Yew WW, Cynamon M, Zhang Y. Emerging drugs for the treatment of tuberculosis. Expert Opin Emerg Drugs 2011;16:1-21.

13. Zumla AI, Gillespie SH, Hoelscher M, et al. New antituberculosis drugs, regimens, and adjunct therapies: needs, advances, and future prospects. Lancet Infect Dis 2014;14:327-340.

14. Sirgel FA, Fourie PB, Donald PR, et al. The early bactericidal activities of rifampin and rifapentine in pulmonary tuberculosis. Am J Respir Crit Care Med 2005;172:128-135.

15. Mitchison DA. The action of antituberculosis drugs in short-course chemotherapy. Tubercle 1985;66:219-225.

16. Mitchison DA. The diagnosis and therapy of tuberculosis during the past 100 years. Am J Respir Crit Care Med 2005;171:699-706.

17. Dorman SE, Goldberg S, Stout JE, et al. Substitution of rifapentine for rifampin during intensive phase treatment of pulmonary tuberculosis: study 29 of the tuberculosis trials consortium. J Infect Dis 2012;206:10301040.

18. Dorman SE, Savic RM, Goldberg S, et al. Daily rifapentine for treatment of pulmonary tuberculosis: a randomized, dose-ranging trial. Am J Respir Crit Care Med 2015;191:333-343.

19. Boeree MJ, Diacon AH, Dawson R, et al. A dose-ranging trial to optimize the dose of rifampin in the treatment of tuberculosis. Am J Respir Crit Care Med 2015;191:1058-1065.

20. Satyaraddi A, Velpandian T, Sharma SK, et al. Correla- tion of plasma anti-tuberculosis drug levels with subsequent development of hepatotoxicity. Int J Tuberc Lung Dis 2014;18:188-195, i-iii.

21. Horne DJ, Royce SE, Gooze L, et al. Sputum monitoring during tuberculosis treatment for predicting outcome: systematic review and meta-analysis. Lancet Infect Dis 2010;10:387-394.

22. Mukamolova GV, Turapov O, Malkin J, Woltmann G, Barer MR. Resuscitation-promoting factors reveal an occult population of tubercle Bacilli in Sputum. Am J Respir Crit Care Med 2010;181:174-180.

23. De Groote MA, Nahid P, Jarlsberg L, et al. Elucidating novel serum biomarkers associated with pulmonary tuberculosis treatment. PLoS One 2013;8:e61002.

24. Garton NJ, Waddell SJ, Sherratt AL, et al. Cytological and transcript analyses reveal fat and lazy persister-like bacilli in tuberculous sputum. PLoS Med 2008;5:e75.

25. Nikolayevskyy V, Miotto P, Pimkina E, et al. Utility of propidium monoazide viability assay as a biomarker for a tuberculosis disease. Tuberculosis (Edinb) 2015;95:179185 .

26. Nuermberger EL, Yoshimatsu T, Tyagi S, et al. Moxifloxacin-containing regimen greatly reduces time to culture conversion in murine tuberculosis. Am J Respir Crit Care Med 2004;169:421-426.

27. Nuermberger EL, Yoshimatsu T, Tyagi S, et al. Moxifloxacin-containing regimens of reduced duration produce a stable cure in murine tuberculosis. Am J Respir Crit Care Med 2004;170:1131-1134.

28. Burman WJ, Goldberg S, Johnson JL, et al. Moxifloxacin versus ethambutol in the first 2 months of treatment for pulmonary tuberculosis. Am J Respir Crit Care Med 2006;174:331-338.

29. Dorman SE, Johnson JL, Goldberg S, et al. Substitution of moxifloxacin for isoniazid during intensive phase treatment of pulmonary tuberculosis. Am J Respir Crit Care Med 2009;180:273-280.

30. Wang JY, Wang JT, Tsai TH, et al. Adding moxifloxacin is associated with a shorter time to culture conversion in pulmonary tuberculosis. Int J Tuberc Lung Dis 2010;14:65-71.

31. Conde MB, Efron A, Loredo C, et al. Moxifloxacin versus ethambutol in the initial treatment of tuberculosis: a double-blind, randomised, controlled phase II trial. Lancet 2009;373:1183-1189.

32. Jawahar MS, Banurekha VV, Paramasivan CN, et al. 
Randomized clinical trial of thrice-weekly 4-month moxifloxacin or gatifloxacin containing regimens in the treatment of new sputum positive pulmonary tuberculosis patients. PLoS One 2013;8:e67030.

33. Gillespie SH, Crook AM, McHugh TD, et al. Fourmonth moxifloxacin-based regimens for drug-sensitive tuberculosis. N Engl J Med 2014;371:1577-1587.

34. Merle CS, Fielding K, Sow OB, et al. A four-month gatifloxacin-containing regimen for treating tuberculosis. N Engl J Med 2014;371:1588-1598.

35. Jindani A, Harrison TS, Nunn AJ, et al. High-dose rifapentine with moxifloxacin for pulmonary tuberculosis. N Engl J Med 2014;371:1599-1608.

36. Velayutham BV, Allaudeen IS, Sivaramakrishnan GN, et al. Sputum culture conversion with moxifloxacin-containing regimens in the treatment of patients with newly diagnosed sputum-positive pulmonary tuberculosis in South India. Clin Infect Dis 2014;59:e142-e149.

37. Rustomjee R, Diacon AH, Allen J, et al. Early bactericidal activity and pharmacokinetics of the diarylquinoline TMC207 in treatment of pulmonary tuberculosis. Antimicrob Agents Chemother 2008;52:2831-2835.

38. Diacon AH, Dawson R, von Groote-Bidlingmaier F, et al. Bactericidal activity of pyrazinamide and clofazimine alone and in combinations with pretomanid and bedaquiline. Am J Respir Crit Care Med 2015;191:943953.

39. Diacon AH, Dawson R, Hanekom M, et al. Early bactericidal activity of delamanid (OPC-67683) in smear-positive pulmonary tuberculosis patients. Int J Tuberc Lung Dis 2011;15:949-954.

40. Diacon AH, Dawson R, Hanekom M, et al. Early bactericidal activity and pharmacokinetics of PA-824 in smear-positive tuberculosis patients. Antimicrob Agents Chemother 2010;54:3402-3407.

41. Diacon AH, Dawson R, du Bois J, et al. Phase II dose-ranging trial of the early bactericidal activity of PA-824. Antimicrob Agents Chemother 2012;56:3027-3031.

42. Diacon AH, Dawson R, von Groote-Bidlingmaier F, et al. 14-Day bactericidal activity of PA-824, bedaquiline, pyrazinamide, and moxifloxacin combinations: a randomised trial. Lancet 2012;380:986-993.

43. Dawson R, Diacon AH, Everitt D, et al. Efficiency and safety of the combination of moxifloxacin, pretomanid (PA-824), and pyrazinamide during the first 8 weeks of antituberculosis treatment: a phase $2 \mathrm{~b}$, open-label, partly randomised trial in patients with drug-susceptible or drug-resistant pulmonary tuberculosis. Lancet 2015;385:1738-1747.

44. Wallis RS, Dawson R, Friedrich SO, et al. Mycobactericidal activity of sutezolid (PNU-100480) in sputum (EBA) and blood (WBA) of patients with pulmonary tuberculosis. PLoS One 2014;9:e94462.

45. Olaru ID, von Groote-Bidlingmaier F, Heyckendorf J, Yew WW, Lange C, Chang KC. Novel drugs against tuberculosis: a clinician's perspective. Eur Respir J 2015;45:1119-1131.

46. Jindani A, Dore CJ, Mitchison DA. Bactericidal and sterilizing activities of antituberculosis drugs during the first 14 days. Am J Respir Crit Care Med 2003;167:13481354 .

47. Gunther G, van Leth F, Alexandru S, et al. Multidrug-resistant tuberculosis in Europe, 2010-2011. Emerg Infect Dis 2015;21:409-416.

48. Nijland HM, Ruslami R, Suroto AJ, et al. Rifampicin reduces plasma concentrations of moxifloxacin in patients with tuberculosis. Clin Infect Dis 2007;45:10011007.

49. Heinrich N, Dawson R, du Bois J, et al. Early phase evaluation of SQ109 alone and in combination with rifampicin in pulmonary TB patients. J Antimicrob Chemother 2015;70:1558-1566.

50. Svensson EM, Murray S, Karlsson MO, Dooley KE. Rifampicin and rifapentine significantly reduce concentrations of bedaquiline, a new anti-TB drug. J Antimicrob Chemother 2015;70:1106-1114.

51. Yew WW. Clinically significant interactions with drugs used in the treatment of tuberculosis. Drug Saf 2002;25:111133 .

52. Dooley KE, Park JG, Swindells S, et al. Safety, tolerability, and pharmacokinetic interactions of the antituberculous agent TMC2O7 (bedaquiline) with efavirenz in healthy volunteers: AIDS Clinical Trials Group Study A5267. J Acquir Immune Defic Syndr 2012;59:455-462.

53. Svensson EM, Aweeka F, Park JG, Marzan F, Dooley KE, Karlsson MO. Model-based estimates of the effects of efavirenz on bedaquiline pharmacokinetics and suggested dose adjustments for patients coinfected with HIV and tuberculosis. Antimicrob Agents Chemother 2013;57:2780-2787.

54. Svensson EM, Dooley KE, Karlsson MO. Impact of lopinavir-ritonavir or nevirapine on bedaquiline ex- 
posures and potential implications for patients with tuberculosis-HIV coinfection. Antimicrob Agents Chemother 2014;58:6406-6412.

55. Dooley KE, Kim PS, Williams SD, Hafner R. TB and HIV therapeutics: pharmacology research priorities. AIDS Res Treat 2012;2012:874083.

56. Dooley KE, Luetkemeyer AF, Park JG, et al. Phase I safety, pharmacokinetics, and pharmacogenetics study of the antituberculosis drug PA-824 with concomitant lopinavir-ritonavir, efavirenz, or rifampin. Antimicrob Agents Chemother 2014;58:5245-5252.

57. Shimokawa Y, Sasahara K, Yoda N, Mizuno K, Umehara K. Delamanid does not inhibit or induce cytochrome p450 enzymes in vitro. Biol Pharm Bull 2014;37:17271735 .

58. Jia L, Noker PE, Coward L, Gorman GS, Protopopova $\mathrm{M}$, Tomaszewski JE. Interspecies pharmacokinetics and in vitro metabolism of SQ109. Br J Pharmacol 2006;147:476-485.

59. Reddy VM, Einck L, Andries K, Nacy CA. In vitro interactions between new antitubercular drug candidates SQ109 and TMC207. Antimicrob Agents Chemother 2010;54:2840-2846.

6o. Ahuja SD, Ashkin D, Avendano M, et al. Multidrug resistant pulmonary tuberculosis treatment regimens and patient outcomes: an individual patient data meta-analysis of 9,153 patients. PLoS Med 2012;9:e1001300.

61. Mdluli K, Kaneko T, Upton A. The tuberculosis drug discovery and development pipeline and emerging drug targets. Cold Spring Harb Perspect Med 2015;5:a021154.

62. Falzon D, Jaramillo E, Schunemann HJ, et al. WHO guidelines for the programmatic management of drug-resistant tuberculosis: 2011 update. Eur Respir J 2011;38:516-528.

63. Van Deun A, Maug AK, Salim MA, et al. Short, highly effective, and inexpensive standardized treatment of multidrug-resistant tuberculosis. Am J Respir Crit Care Med 2010;182:684-692.

64. Grosset JH, Tyagi S, Almeida DV, et al. Assessment of clofazimine activity in a second-line regimen for tuberculosis in mice. Am J Respir Crit Care Med 2013;188:608-612.

65. Padayatchi N, Gopal M, Naidoo R, et al. Clofazimine in the treatment of extensively drug-resistant tuberculosis with HIV coinfection in South Africa: a retrospective cohort study. J Antimicrob Chemother 2014;69:3103-3107.
66. Tang S, Yao L, Hao X, et al. Clofazimine for the treatment of multidrug-resistant tuberculosis: prospective, multicenter, randomized controlled study in China. Clin Infect Dis 2015;60:1361-1367.

67. Aung KJ, Van Deun A, Declercq E, et al. Successful '9-month Bangladesh regimen' for multidrug-resistant tuberculosis among over 500 consecutive patients. Int J Tuberc Lung Dis 2014;18:1180-1187.

68. Yew WW, Lange C. Fluoroquinolone resistance in Mycobacterium tuberculosis: what have we learnt? Int J Tuberc Lung Dis 2014;18:1-2.

69. Nunn AJ, Rusen ID, Van Deun A, et al. Evaluation of a standardized treatment regimen of anti-tuberculosis drugs for patients with multi-drug-resistant tuberculosis (STREAM): study protocol for a randomized controlled trial. Trials 2014;15:353.

70. Dooley KE, Obuku EA, Durakovic N, et al. World Health Organization group 5 drugs for the treatment of drug-resistant tuberculosis: unclear efficacy or untapped potential? J Infect Dis 2013;207:1352-1358.

71. Cox H, Ford N. Linezolid for the treatment of complicated drug-resistant tuberculosis: a systematic review and meta-analysis. Int J Tuberc Lung Dis 2012;16:447454 .

72. Sotgiu G, Centis R, D'Ambrosio L, et al. Efficacy, safety and tolerability of linezolid containing regimens in treating MDR-TB and XDR-TB: systematic review and meta-analysis. Eur Respir J 2012;40:1430-1442.

73. Koh WJ, Kang YR, Jeon K, et al. Daily $300 \mathrm{mg}$ dose of linezolid for multidrug-resistant and extensively drug-resistant tuberculosis: updated analysis of 51 patients. J Antimicrob Chemother 2012;67:1503-1507.

74. Lee M, Lee J, Carroll MW, et al. Linezolid for treatment of chronic extensively drug-resistant tuberculosis. N Engl J Med 2012;367:1508-1518.

75. Chang KC, Yew WW, Cheung SW, et al. Can intermittent dosing optimize prolonged linezolid treatment of difficult multidrug-resistant tuberculosis? Antimicrob Agents Chemother 2013:57:3445-3449.

76. Chang KC, Yew WW, Tam CM, Leung CC. WHO group 5 drugs and difficult multidrug-resistant tuberculosis: a systematic review with cohort analysis and meta-analysis. Antimicrob Agents Chemother 2013;57:4097-4104.

77. Dey T, Brigden G, Cox H, Shubber Z, Cooke G, Ford N. Outcomes of clofazimine for the treatment of drug-resistant tuberculosis: a systematic review and meta-anal- 
ysis. J Antimicrob Chemother 2013;68:284-293.

78. Gopal M, Padayatchi N, Metcalfe JZ, O'Donnell MR. Systematic review of clofazimine for the treatment of drug-resistant tuberculosis. Int J Tuberc Lung Dis 2013;17:1001-1007.

79. Payen MC, De Wit S, Martin C, et al. Clinical use of the meropenem-clavulanate combination for extensively drug-resistant tuberculosis. Int J Tuberc Lung Dis 2012;16:558-560.

8o. De Lorenzo S, Alffenaar JW, Sotgiu G, et al. Efficacy and safety of meropenem-clavulanate added to linezolid-containing regimens in the treatment of MDR-/ XDR-TB. Eur Respir J 2013;41:1386-1392.

81. Diacon AH, Pym A, Grobusch M, et al. The diarylquinoline TMC2O7 for multidrug-resistant tuberculosis. N Engl J Med 2009;360:2397-2405.

82. Diacon AH, Donald PR, Pym A, et al. Randomized pilot trial of eight weeks of bedaquiline (TMC207) treatment for multidrug-resistant tuberculosis: long-term outcome, tolerability, and effect on emergence of drug resistance. Antimicrob Agents Chemother 2012;56:3271-3276.

83. Diacon AH, Pym A, Grobusch MP, et al. Multidrug-resistant tuberculosis and culture conversion with bedaquiline. N Engl J Med 2014;371:723-732.

84. Guglielmetti L, Le Du D, Jachym M, et al. Compassionate use of bedaquiline for the treatment of multidrug-resistant and extensively drug-resistant tuberculosis: interim analysis of a French cohort. Clin Infect Dis 2015;60:188-194.

85. Gler MT, Skripconoka V, Sanchez-Garavito E, et al. Delamanid for multidrug-resistant pulmonary tuberculosis. N Engl J Med 2012;366:2151-2160.

86. Skripconoka V, Danilovits M, Pehme L, et al. Delamanid improves outcomes and reduces mortality in multidrug-resistant tuberculosis. Eur Respir J 2013;41:13931400.

87. Wells CD, Gupta R, Hittel N, Geiter LJ. Long-term mortality assessment of multidrug-resistant tuberculosis patients treated with delamanid. Eur Respir J 2015;45:1498-1501.

88. Isaakidis P, Varghese B, Mansoor H, et al. Adverse events among HIV/MDR-TB co-infected patients receiving antiretroviral and second line anti-TB treatment in Mumbai, India. PLoS One 2012;7:e40781.

89. Daskapan A, de Lange WC, Akkerman OW, et al. The role of therapeutic drug monitoring in individualised drug dosage and exposure measurement in tuberculosis and HIV co-infection. Eur Respir J 2015;45:569-571.

90. Sotgiu G, Alffenaar JW, Centis R, et al. Therapeutic drug monitoring: how to improve drug dosage and patient safety in tuberculosis treatment. Int J Infect Dis 2015;32:101-104.

91. Horsburgh CR Jr, Haxaire-Theeuwes M, Lienhardt C, et al. Compassionate use of and expanded access to new drugs for drug-resistant tuberculosis. Int J Tuberc Lung Dis 2013;17:146-152.

92. Huang TS, Liu YC, Sy CL, Chen YS, Tu HZ, Chen BC. In vitro activities of linezolid against clinical isolates of Mycobacterium tuberculosis complex isolated in Taiwan over 10 years. Antimicrob Agents Chemother 2008;52:2226-2227.

93. Zhang Z, Pang Y, Wang Y, Liu C, Zhao Y. Beijing genotype of Mycobacterium tuberculosis is significantly associated with linezolid resistance in multidrug-resistant and extensively drug-resistant tuberculosis in China. Int J Antimicrob Agents 2014;43:231-235.

94. Hartkoorn RC, Uplekar S, Cole ST. Cross-resistance between clofazimine and bedaquiline through upregulation of MmpL5 in Mycobacterium tuberculosis. Antimicrob Agents Chemother 2014;58:2979-2981.

95. Andries K, Villellas C, Coeck N, et al. Acquired resistance of Mycobacterium tuberculosis to bedaquiline. PLoS One 2014;9:e102135.

96. Somoskovi A, Bruderer V, Homke R, Bloemberg GV, Bottger EC. A mutation associated with clofazimine and bedaquiline cross-resistance in MDR-TB following bedaquiline treatment. Eur Respir J 2015;45:554-557.

97. Gupta S, Cohen KA, Winglee K, Maiga M, Diarra B, Bishai WR. Efflux inhibition with verapamil potentiates bedaquiline in Mycobacterium tuberculosis. Antimicrob Agents Chemother 2014;58:574-576.

98. Gupta S, Tyagi S, Bishai WR. Verapamil increases the bactericidal activity of bedaquiline against Mycobacterium tuberculosis in a mouse model. Antimicrob Agents Chemother 2015;59:673-676.

99. Dharmadhikari AS, Kabadi M, Gerety B, Hickey AJ, Fourie PB, Nardell E. Phase I, single-dose, dose-escalating study of inhaled dry powder capreomycin: a new approach to therapy of drug-resistant tuberculosis. Antimicrob Agents Chemother 2013;57:2613-2619.

100. Park JH, Jin HE, Kim DD, Chung SJ, Shim WS, Shim CK. Chitosan microspheres as an alveolar macrophage 
delivery system of ofloxacin via pulmonary inhalation. Int J Pharm 2013;441(1-2):562-569.

101. Verma RK, Germishuizen WA, Motheo MP, et al. Inhaled microparticles containing clofazimine are efficacious in treatment of experimental tuberculosis in mice. Antimicrob Agents Chemother 2013;57:1050-1052. 102. Sung JC, Garcia-Contreras L, Verberkmoes JL, et al. Dry powder nitroimidazopyran antibiotic PA-824 aerosol for inhalation. Antimicrob Agents Chemother 2009;53:13381343. 